\title{
DIRECT AND INVERSE THEOREMS FOR MULTIVARIATE BERNSTEIN-SCHURER-STANCU OPERATORS
}

\author{
MEHMET ALI ÖZARSLAN AND TUBA VEDI
}

Received 18 April, 2014

\begin{abstract}
In this paper, we introduce the multivariate Bernstein-Schurer-Stancu operators. Then, we state the Volkov-type theorem and investigate the order of convergence by means of modulus of continuity and by Lipschitz class functionals. Moreover, the inverse theorems are studied for the multivariate Stancu variant of Bernstein operators.
\end{abstract}

2010 Mathematics Subject Classification: 41A25; 41A36

Keywords: multivariate Bernstein-Schurer-Stancu operators, modulus of continuity, direct theorem, inverse theorems

\section{INTRODUCTION}

The celebrated Bernstein polynomials [16]

$$
B_{n}(f ; x)=\sum_{k=0}^{n} f\left(\frac{k}{n}\right)\left(\begin{array}{l}
n \\
k
\end{array}\right) x^{k}(1-x)^{k}, \quad x \in[0,1]
$$

have been the most attractive approximating operators which were studied by various mathematicians and have many applications in mathematics, physics, engineering, economy, etc (see [3], [4], [7], [8], [9], [10],[11], [12], [14], [15], [17], [18], [19], [20], [21], [23]) .

In the year 1962, Schurer [22] defined the Schurer operators as:

$$
B_{n, p}(f ; x)=\sum_{k=0}^{n+p} f\left(\frac{k}{n}\right)\left(\begin{array}{c}
n+p \\
k
\end{array}\right) x^{k}(1-x)^{n+p-k}, \quad x \in[0,1] .
$$

We should notice that if $p=0$ in (1.1), we have $B_{n, 0}(f ; x)=B_{n}(f ; x)$.

On the other hand, Barbosu [1] estimated the Schurer-Stancu operators as:

$$
\left(\tilde{S}_{n, p}^{(\alpha, \beta)} f\right)(x)=\sum_{k=0}^{n+p} f\left(\frac{k+\alpha}{n+\beta}\right)\left(\begin{array}{c}
n+p \\
k
\end{array}\right) x^{k}(1-x)^{n+p-k}, \quad x \in[0,1]
$$


where $\alpha$ and $\beta$ are real parameters such that $0 \leq \alpha \leq \beta$ and $p$ is a fixed non-negative integer.

Letting $\alpha=\beta=0$ in (1.2), we have

$$
\left(\tilde{S}_{n, p}^{(0,0)} f\right)(x)=B_{n, p}(f ; x) .
$$

Afterwards, Barbosu introduced the bivariate operators of Schurer-Stancu type in [2] as:

$$
\begin{aligned}
& \left(\tilde{S}_{m, p, n, q}^{\left(\alpha_{1}, \alpha_{2}, \beta_{1}, \beta_{2}\right)} f\right)(x, y)=\sum_{k=0}^{n+p} \sum_{j=0}^{m+q} f\left(\frac{k+\alpha_{1}}{n+\beta_{1}}, \frac{j+\alpha_{2}}{m+\beta_{2}}\right)\left(\begin{array}{c}
n+p \\
k
\end{array}\right)\left(\begin{array}{c}
m+q \\
j
\end{array}\right) \\
& \times x^{k}(1-x)^{n+p-k} y^{k}(1-y)^{m+q-j},
\end{aligned}
$$

where $m, n \in \mathbb{N}, p, q \in \mathbb{N}_{0}$ and $\alpha_{1}, \beta_{1}, \alpha_{2}, \beta_{2}$ are real numbers which satisfy the conditions $0 \leq \alpha_{1} \leq \beta_{1}$ and $0 \leq \alpha_{2} \leq \beta_{2}$ and $(x, y) \in[0,1] \times[0,1]$. Then he obtained the Volkov-theorem (bivariate Bohman-Korovkin theorem) and studied the order of convergence of these operators by means of the first modulus of continuity for two variables functions. It should be noted that, taking $p=q=0$ in (1.3), we get the operators investigated in [5].

In 2008, Büyükyazıcı and İbikli introduced the multivariate generalized Bernstein polynomials [6] as:

$$
B_{n}\left(f ; x_{1}, \ldots, x_{m}\right):=\sum_{k_{1}, \ldots, k_{m}=0}^{n} f\left(\frac{k_{1}}{b_{n}}, \cdots, \frac{k_{m}}{b_{n}}\right) \prod_{j=1}^{m}\left[\left(\begin{array}{c}
n \\
k_{j}
\end{array}\right) x_{j}^{k_{j}}\left(1-x_{j}\right)^{n-k_{j}}\right]
$$

where $\left(b_{n}\right)$ is a sequence of positive numbers such that $\lim _{n \rightarrow \infty} b_{n}=\infty$ and $\lim _{n \rightarrow \infty} \frac{b_{n}}{n}=$ 1. Then, they studied direct and inverse theorems for the multivariate generalized Bernstein operators.

We organize the paper in the following way:

In section two, the multivariate Bernstein-Schurer-Stancu operators are introduced and the moments of the operators are calculated. In section three, the Volkov-type theorems are obtained. In section four, we give quantitative results for the error in terms of the modulus of continuiy and the Lipschitz class functionals. In section five, we study inverse theorems of the multivariate Bernstein-Schurer-Stancu operators.

\section{CONSTRUCTION OF THE OPERATORS}

We introduce the multivariate Bernstein-Schurer-Stancu operators by

$B_{n, p}^{\left(\alpha_{1}, \ldots, \alpha_{m}\right)}\left(f ; x_{1}, \ldots, x_{m}\right)$ 


$$
:=\sum_{k_{1}, \ldots, k_{m}=0}^{n+p} f\left(\frac{k_{1}+\alpha_{1}}{b_{n}}, \cdots, \frac{k_{m}+\alpha_{m}}{b_{n}}\right) \prod_{j=1}^{m}\left[\left(\begin{array}{c}
n+p \\
k_{j}
\end{array}\right) x_{j}^{k_{j}}\left(1-x_{j}\right)^{n+p-k_{j}}\right]
$$

where $\boldsymbol{\alpha}=\left(\alpha_{1}, \ldots, \alpha_{m}\right)$ be $m$-tuples real parameters satisfying the conditions $\alpha_{i} \geq 0$ $(i=1, \ldots, m), f$ be a continuous function on $D^{p}:=\bigotimes_{j=1}^{m}[0, p+1]$, for fixed $p \in \mathbb{N}_{0}$ and $\left(b_{n}\right)$ is a sequence of positive real numbers satisfying

$\left(1^{\circ}\right)$ For each $n \in \mathbb{N}, n+\max \left\{\alpha_{1}, \cdots, \alpha_{m}\right\} \leq b_{n}$,

$\left(2^{\circ}\right) \lim _{n \rightarrow \infty} \frac{b_{n}}{n}=1$.

It is clear that $B_{n, p}^{\left(\alpha_{1}, \ldots, \alpha_{m}\right)}: C_{\mathbb{R}^{m}}\left(D^{p}\right) \rightarrow C_{\mathbb{R}^{m}}\left(D^{0}\right)$. Note that, if we choose $p=0$ and $\alpha=\left(\alpha_{1}, \ldots, \alpha_{m}\right)=(0, \ldots, 0)$ in (2.1), we get the operators in (1.4).

In giving the approximation properties of the operators $B_{n, p}^{\left(\alpha_{1}, \ldots, \alpha_{m}\right)}\left(f ; x_{1}, \ldots, x_{m}\right)$, we need the following lemma:

Lemma 1. Let $B_{n, p}^{\left(\alpha_{1}, \ldots, \alpha_{m}\right)}\left(f ; x_{1}, \ldots, x_{m}\right)$ be given in (2.1). The first few moments of the operators are

$$
\begin{aligned}
& \text { i) } B_{n, p}^{\left(\alpha_{1}, \ldots, \alpha_{m}\right)}\left(1 ; x_{1}, \ldots, x_{m}\right)=1, \\
& \text { ii) } B_{n, p}^{\left(\alpha_{1}, \ldots, \alpha_{m}\right)}\left(t_{j} ; x_{1}, \ldots, x_{m}\right)=\frac{n+p}{b_{n}} x_{j}+\frac{\alpha_{j}}{b_{n}}, \quad(j=1,2, \ldots, m), \\
& \text { ii i }) B_{n, p}^{\left(\alpha_{1}, \ldots, \alpha_{m}\right)}\left(|\mathbf{t}|^{2} ; x_{1}, \ldots, x_{m}\right) \\
& \quad=\frac{(n+p)^{2}}{b_{n}^{2}}|\mathbf{x}|^{2}-\frac{n+p}{b_{n}^{2}}|\mathbf{x}|^{2}+\frac{|\boldsymbol{\alpha}|^{2}}{b_{n}^{2}}+\frac{n+p}{b_{n}^{2}} \sum_{j=0}^{m}\left(2 \alpha_{j}+1\right) x_{j} \\
& \text { iv) } B_{n, p}^{\left(\alpha_{1}, \ldots, \alpha_{m}\right)}\left((\mathbf{t}-\mathbf{x})^{2} ; x_{1}, \ldots, x_{m}\right)=\left(\left(\frac{n+p}{b_{n}}-1\right)^{2}-\frac{n+p}{b_{n}^{2}}\right)|\mathbf{x}|^{2} \\
& +\sum_{j=1}^{m}\left[\frac{n+p}{b_{n}^{2}}\left(2 \alpha_{j}+1\right)-2 \frac{\alpha_{j}}{b_{n}}\right] x_{j}+\frac{|\boldsymbol{\alpha}|^{2}}{b_{n}^{2}}(j=1,2, \ldots, m), \\
& \text { where }|\mathbf{x}|=\sum_{i=1}^{m} x_{i},|\mathbf{t}|=\sum_{i=1}^{m} t_{i},|\boldsymbol{\alpha}|=\sum_{i=1}^{m} \alpha_{i} .
\end{aligned}
$$

Proof. $i)$ It is clear that $B_{n, p}^{\left(\alpha_{1}, \ldots, \alpha_{m}\right)}\left(1 ; x_{1}, \ldots, x_{m}\right)=1$.

ii) For the proof of (ii), we have

$$
\begin{gathered}
B_{n, p}^{\left(\alpha_{1}, \ldots, \alpha_{m}\right)}\left(t_{j} ; x_{1}, \ldots, x_{m}\right) \\
=\sum_{k_{1}, \ldots, k_{m}=0}^{n+p}\left(\frac{k_{j}+\alpha_{j}}{b_{n}}\right) \prod_{i=1}^{m}\left[\left(\begin{array}{c}
n+p \\
k_{i}
\end{array}\right) x_{i}^{k_{i}}\left(1-x_{i}\right)^{n+p-k_{i}}\right] \\
=\frac{n+p}{b_{n}} \sum_{k_{1}, \ldots, k_{m}=0}^{n+p} \frac{k_{j}}{n+p} \prod_{i=1}^{m}\left[\left(\begin{array}{c}
n+p \\
k_{i}
\end{array}\right) x_{i}^{k_{i}}\left(1-x_{i}\right)^{n+p-k_{i}}\right]+\frac{\alpha_{j}}{b_{n}} .
\end{gathered}
$$


Then after some straightforward calculations, we obtain the result directly.

i i i ) Using the operator $B_{n, p}^{\left(\alpha_{1}, \ldots, \alpha_{m}\right)}\left(f ; x_{1}, \ldots, x_{m}\right)$, we have

$$
\begin{aligned}
& B_{n, p}^{\left(\alpha_{1}, \ldots, \alpha_{m}\right)}\left(|\mathbf{t}|^{2} ; x_{1}, \ldots, x_{m}\right) \\
= & \sum_{k_{1}, \ldots, k_{m}=0}^{n+p} \sum_{j=1}^{m}\left(\frac{k_{j}+\alpha_{j}}{b_{n}}\right)^{2} \prod_{i=1}^{m}\left[\left(\begin{array}{c}
n+p \\
k_{i}
\end{array}\right) x_{i}^{k_{i}}\left(1-x_{i}\right)^{n+p-k_{i}}\right] \\
= & \sum_{k_{1}, \ldots, k_{m}=0}^{n+p} \sum_{j=1}^{m}\left(\frac{k_{j}^{2}+2 k_{j} \alpha_{j}+\alpha_{j}^{2}}{b_{n}^{2}}\right) \prod_{i=1}^{m}\left[\left(\begin{array}{c}
n+p \\
k_{i}
\end{array}\right) x_{i}^{k_{i}}\left(1-x_{i}\right)^{n+p-k_{i}}\right] \\
= & \frac{(n+p)^{2}}{b_{n}^{2}} \sum_{j=1}^{m} \sum_{k_{1}, \ldots, k_{m}=0}^{n+p}\left(\frac{k_{j}^{2}}{(n+p)^{2}}\right) \prod_{i=1}^{m}\left[\left(\begin{array}{c}
n+p \\
k_{i}
\end{array}\right) x_{i}^{k_{i}}\left(1-x_{i}\right)^{n+p-k_{i}}\right] \\
+ & \frac{2(n+p)}{b_{n}^{2}} \sum_{j=1}^{m} \alpha_{j} \sum_{k_{1}, \ldots, k_{m}=0}^{n+p}\left(\frac{k_{j}}{n+p}\right) \prod_{i=1}^{m}\left[\left(\begin{array}{c}
n+p \\
k_{i}
\end{array}\right) x_{i}^{k_{i}}\left(1-x_{i}\right)^{n+p-k_{i}}\right]+\frac{|\boldsymbol{\alpha}|^{2}}{b_{n}^{2}} .
\end{aligned}
$$

Since $|\mathbf{x}|=\sum_{i=1}^{m} x_{i}$ and $|\boldsymbol{\alpha}|=\sum_{i=1}^{m} \alpha_{i}$ then we have

$$
\begin{aligned}
& B_{n, p}^{\left(\alpha_{1}, \ldots, \alpha_{m}\right)}\left(|\mathbf{t}|^{2} ; x_{1}, \ldots, x_{m}\right) \\
& =\frac{(n+p)^{2}}{b_{n}^{2}}|\mathbf{x}|^{2}-\frac{n+p}{b_{n}^{2}}|\mathbf{x}|^{2}+\frac{|\boldsymbol{\alpha}|^{2}}{b_{n}^{2}}+\frac{n+p}{b_{n}^{2}} \sum_{j=1}^{m}\left(2 \alpha_{j}+1\right) x_{j} .
\end{aligned}
$$

Then, the proof is completed.

iv) Using (i), (ii) and (iii) we have

$$
\begin{array}{r}
B_{n, p}^{\left(\alpha_{1}, \ldots, \alpha_{m}\right)}\left((\mathbf{t}-\mathbf{x})^{2} ; x_{1}, \ldots, x_{m}\right)=B_{n, p}^{\left(\alpha_{1}, \ldots, \alpha_{m}\right)}\left(|\mathbf{t}|^{2} ; x_{1}, \ldots, x_{m}\right) \\
-2 B_{n, p}^{\left(\alpha_{1}, \ldots, \alpha_{m}\right)}\left(\sum_{j=1}^{m} x_{j} t_{j} ; x_{1}, \ldots, x_{m}\right)+B_{n, p}^{\left(\alpha_{1}, \ldots, \alpha_{m}\right)}\left(|\mathbf{x}|^{2} ; x_{1}, \ldots, x_{m}\right)
\end{array}
$$

Hence, we get the desired result.

\section{VOLKOV-TYPE APPROXIMATION}

In this section, the Volkov-type approximation theorem is proved for the operators $B_{n, p}^{\left(\alpha_{1}, \ldots, \alpha_{m}\right)}\left(f ; x_{1}, \ldots, x_{m}\right)$. Let $C_{\mathbb{R}^{m}}\left(D^{0}\right)$ be a linear normed space endowed with the following norm

$$
\|g\|_{C_{\mathbb{R}} m}\left(D^{0}\right)=\max _{\left(x_{1}, x_{2}, \ldots, x_{m}\right) \in D^{0}}\left|g\left(x_{1}, x_{2}, \ldots, x_{m}\right)\right| .
$$


We will use the following Volkov-type approximation theorem to give our main results of this section.

Theorem 1 ([13]). Let $T_{n}: C_{\mathbb{R}^{m}}\left(D^{0}\right) \rightarrow C_{\mathbb{R}^{m}}\left(D^{0}\right)$ be a sequence of linear positive operators. If the following $(m+2)$ conditions are satisfied

$$
\begin{aligned}
\lim _{n \rightarrow \infty}\left\|T_{n}(1 ; x)-1\right\|_{C_{\mathbb{R}}\left(D^{0}\right)} & =0, \\
\lim _{n \rightarrow \infty}\left\|T_{n}\left(t_{j} ; x\right)-x_{j}\right\|_{C_{\mathbb{R}}\left(D^{0}\right)} & =0, \quad(j=1,2, \ldots, m) \\
\lim _{n \rightarrow \infty}\left\|T_{n}\left(|\mathbf{t}|^{2} ; x\right)-|\mathbf{x}|^{2}\right\|_{C_{\mathbb{R}}\left(D^{0}\right)} & =0,
\end{aligned}
$$

where $\mathbf{t}=\left(t_{1}, \ldots, t_{m}\right), \mathbf{x}=\left(x_{1}, \ldots, x_{m}\right)$, then for any function $f \in C_{\mathbb{R}^{m}}\left(D^{0}\right)$, we have

$$
\left\|T_{n}(f ; \cdot)-f(\cdot)\right\|_{\mathbb{R}^{m}\left(D^{0}\right)}=0, n \rightarrow \infty .
$$

Note that, similar theorem can be stated for the operator $T_{n}: C_{\mathbb{R}^{m}}\left(D^{p}\right) \rightarrow C_{\mathbb{R}^{m}}\left(D^{0}\right)$.

Theorem 2. Let the sequence of real numbers $\left(b_{n}\right)$ satisfies $\left(1^{\circ}\right)$ and $\left(2^{\circ}\right)$. For all $f \in C_{\mathbb{R}^{m}}\left(D^{p}\right), B_{n, p}^{\left(\alpha_{1}, \ldots, \alpha_{m}\right)}\left(f ; x_{1}, \ldots, x_{m}\right)$ is uniformly convergent to $f$ as $n \rightarrow \infty$.

Proof. Using Lemma 1 and taking into account $\left(1^{\circ}\right)$ and $\left(2^{\circ}\right)$, we have

$$
\begin{aligned}
\left\|B_{n, p}^{\left(\alpha_{1}, \ldots, \alpha_{m}\right)}(1 ; x)-1\right\|_{C_{\mathbb{R}}\left(D^{0}\right)} & =0, \\
\lim _{n \rightarrow \infty}\left\|B_{n, p}^{\left(\alpha_{1}, \ldots, \alpha_{m}\right)}\left(t_{j} ; x\right)-x_{j}\right\|_{C_{\mathbb{R} m}\left(D^{0}\right)} & =0,(j=1,2, \ldots, m) \\
\lim _{n \rightarrow \infty}\left\|B_{n, p}^{\left(\alpha_{1}, \ldots, \alpha_{m}\right)}\left(|\mathbf{t}|^{2} ; x\right)-|\mathbf{x}|^{2}\right\|_{C_{\mathbb{R}}\left(D^{0}\right)} & =0 .
\end{aligned}
$$

Thus, the conditions (3.1), (3.2) and (3.3) are satisfied and the proof follows from Theorem 1.

Remark 1. If we choose $m=2$ in (2.1), the multivariate Bernstein-Schurer-Stancu operators for $f\left(x_{1}, x_{2}\right)$ have the following form:

$$
\begin{aligned}
B_{n, p}^{\left(\alpha_{1}, \alpha_{2}\right)}\left(f ; x_{1}, x_{2}\right) & :=\sum_{k_{1}=0}^{n+p} \sum_{k_{2}=0}^{n+p} f\left(\frac{k_{1}+\alpha_{1}}{b_{n}}, \frac{k_{2}+\alpha_{2}}{b_{n}}\right)\left(\begin{array}{c}
n+p \\
k_{1}
\end{array}\right)\left(\begin{array}{c}
n+p \\
k_{2}
\end{array}\right) \\
& \times x_{1}^{k_{1}}\left(1-x_{1}\right)^{n+p-k_{1}} x_{2}^{k_{2}}\left(1-x_{2}\right)^{n+p-k_{2}} .
\end{aligned}
$$

Furthermore, we set the following,

$$
\begin{aligned}
f\left(x_{1}, x_{2}\right) & =\cos \left(x_{1} x_{2}\right), \\
b_{n} & =1 /(\log (n+p+1)-\log (n+p)) \\
n & =5,10,30,60,80,100,
\end{aligned}
$$




$$
\begin{aligned}
p & =1,2,3,4,5,6, \\
\alpha_{1} & =\alpha_{2}=\frac{1}{100}, \frac{1}{90}, \frac{1}{80}, \frac{1}{70}, \frac{1}{60}, \frac{1}{50},
\end{aligned}
$$

in (3.4). According to the above parameters, the convergence of $B_{n, p}^{\left(\alpha_{1}, \alpha_{2}\right)}\left(f ; x_{1}, x_{2}\right)$ to $f$ is shown in Figure 1.

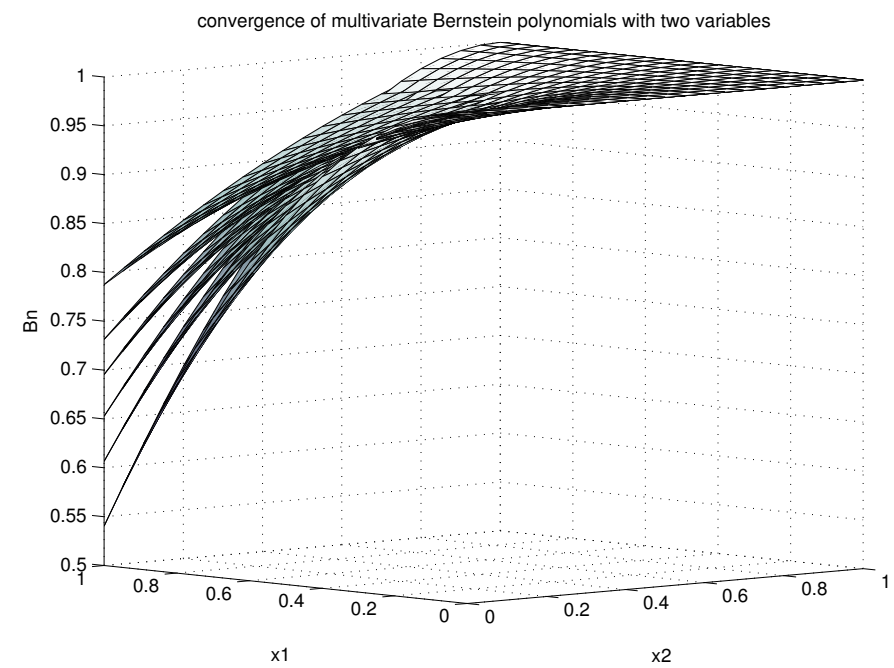

FIGURE 1. The convergence of multivariable Bernstein-SchurerStacu operators with two variables

Remark 2. If we choose $m=3$ in (2.1), the multivariate Bernstein-Schurer-Stancu operators for $f\left(x_{1}, x_{2}, x_{3}\right)$ have the following form:

$$
\begin{aligned}
& B_{n, p}^{\left(\alpha_{1}, \alpha_{2}, \alpha_{3}\right)}\left(f ; x_{1}, x_{2}, x_{3}\right) \\
& :=\sum_{k_{1}=0}^{n+p} \sum_{k_{2}=0}^{n+p} \sum_{k_{3}=0}^{n+p} f\left(\frac{k_{1}+\alpha_{1}}{b_{n}}, \frac{k_{2}+\alpha_{2}}{b_{n}}, \frac{k_{3}+\alpha_{3}}{b_{n}}\right)\left(\begin{array}{c}
n+p \\
k_{1}
\end{array}\right)\left(\begin{array}{c}
n+p \\
k_{2}
\end{array}\right)\left(\begin{array}{c}
n+p \\
k_{3}
\end{array}\right) \\
& \times x_{1}^{k_{1}}\left(1-x_{1}\right)^{n+p-k_{1}} x_{2}^{k_{2}}\left(1-x_{2}\right)^{n+p-k_{2}} x_{3}^{k_{3}}\left(1-x_{3}\right)^{n+p-k_{3}} .
\end{aligned}
$$

Moreover, let us choose

$$
\begin{aligned}
f\left(x_{1}, x_{2}, x_{3}\right) & =\left(x_{1}-\frac{1}{3}\right)^{2}+\left(x_{2}-\frac{1}{3}\right)^{2}+\left(x_{3}-\frac{1}{3}\right)^{2}-\frac{1}{9}, \\
b_{n} & =1 /(\log (n+p+1)-\log (n+p)),
\end{aligned}
$$




$$
\begin{aligned}
n & =5,30,60,80, \\
p & =1, \\
\alpha_{1} & =\alpha_{2}=\frac{1}{100}, \frac{1}{90}, \frac{1}{80}, \frac{1}{70}
\end{aligned}
$$

in (3.5). According to the above parameters, the convergence of $B_{n, p}^{\left(\alpha_{1}, \alpha_{2}, \alpha_{3}\right)}\left(f ; x_{1}, x_{2}, x_{3}\right)$ to $f$ is illustrated in Figure 2.
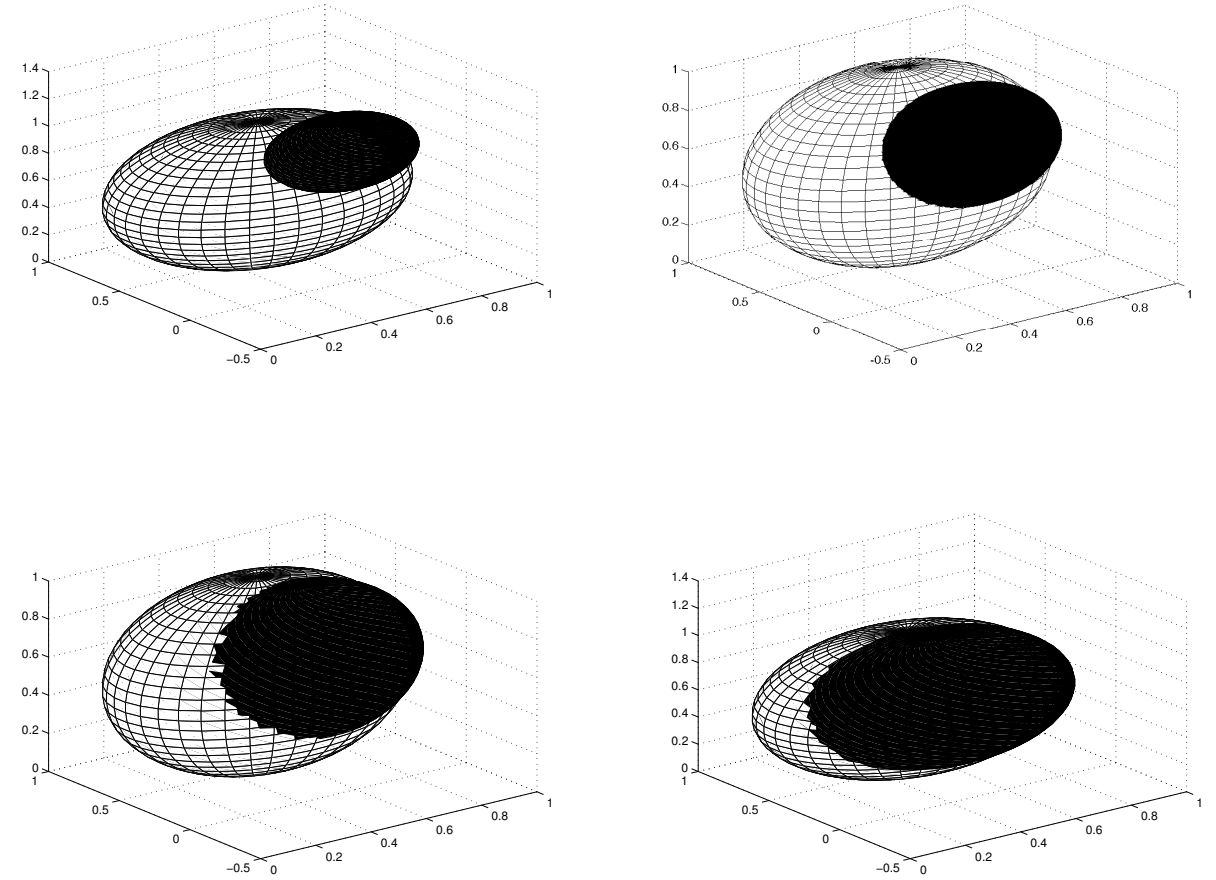

FIGURE 2. The convergence of multivariable Bernstein-SchurerStancu operators with three variables

\section{RATE OF CONVERGENCE}

In this section, we study the order of convergence of multivariate BernsteinSchurer-Stancu operators by means of modulus of continuity and Lipschitz class functionals.

Firstly, let us recall the definitions of modulus of continuity and partial modulus of continuity, recpectively. 
The full modulus of continuity of the function $f \in C_{\mathbb{R}^{m}}\left(D^{p}\right)$ is defined by

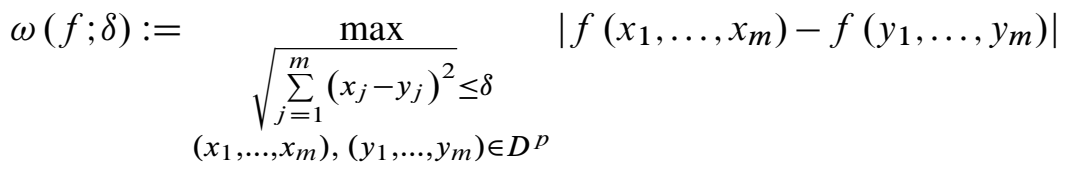

where $f \in C_{\mathbb{R}^{m}}\left(D^{p}\right)$ and $\delta>0$. For each fixed $i=1, \ldots, m$, the partial modulus of continuity of $f$ with respect to $x_{i}$ is defined by

$$
\begin{aligned}
& \omega^{(i)}(f ; \delta):=\max _{0 \leq x_{1}, \ldots, x_{m} \leq 1} \max _{\substack{\left|x_{i}-y_{i}\right| \leq \delta \\
x_{i}, y_{i} \in[0,1+p]}} \\
& \mid f\left(x_{1}, \ldots, x_{i-1}, x_{i}, x_{i+1, \ldots, x_{m}}\right)-f\left(x_{1}, \ldots, x_{i-1}, y_{i}, x_{\left.i+1, \ldots, x_{m}\right) \mid .}\right.
\end{aligned}
$$

Furthermore, it is well known that

$$
\lim _{\delta \rightarrow 0} \omega(f ; \delta)=0
$$

and for any $\lambda>0$,

$$
\omega(f ; \lambda \delta) \leq(\lambda+1) \omega(f ; \delta) .
$$

Note that the same properties are satisfied by partial modulus of continuities. Our quantitative results in terms of full and partial modulus of continuies are presented in the following theorem.

Theorem 3. Let $f \in C_{\mathbb{R}^{m}}\left(D^{p}\right)$, then for all $\mathbf{x}=\left(x_{1}, \ldots, x_{m}\right) \in D^{0}$, the following inequalities

$$
\begin{aligned}
& \text { i) }\left|B_{n, p}^{\left(\alpha_{1}, \ldots, \alpha_{m}\right)}(f ; \mathbf{x})-f(\mathbf{x})\right| \leq 2 \omega\left(f ; \sqrt{\delta_{n}(\mathbf{x})}\right), \\
& \text { ii) }\left|B_{n, p}^{\left(\alpha_{1}, \ldots, \alpha_{m}\right)}(f ; \mathbf{x})-f(\mathbf{x})\right| \leq 2 \sum_{i=1}^{m} \omega^{(i)}\left(f ; \sqrt{\delta_{n}^{(i)}\left(x_{i}\right)}\right)
\end{aligned}
$$

holds, where

$$
=\left(\left(\frac{n+p}{b_{n}}-1\right)^{2}-\frac{n+p}{b_{n}^{2}}\right)|\mathbf{x}|^{2}+\sum_{j=1}^{m}\left[\frac{n+p}{b_{n}^{2}}\left(2 \alpha_{j}+1\right)-2 \frac{\alpha_{j}}{b_{n}}\right] x_{j}+\frac{|\boldsymbol{\alpha}|^{2}}{b_{n}^{2}}
$$

and

$$
\delta_{n}^{(i)}\left(x_{i}\right)=\left(\left(\frac{n+p}{b_{n}}-1\right)^{2}-\frac{n+p}{b_{n}^{2}}\right) x_{i}^{2}+\left[\frac{n+p}{b_{n}^{2}}\left(2 \alpha_{i}+1\right)-2 \frac{\alpha_{i}}{b_{n}}\right] x_{i}+\frac{\alpha_{i}^{2}}{b_{n}^{2}} .
$$

Proof. i) From (2.1) we get

$$
B_{n, p}^{\left(\alpha_{1}, \ldots, \alpha_{m}\right)}(f ; \mathbf{x})-f(\mathbf{x})
$$




$$
\begin{array}{r}
=\sum_{k_{1}, \ldots, k_{m}=0}^{n+p}\left(f\left(\frac{k_{1}+\alpha_{1}}{b_{n}}, \cdots, \frac{k_{m}+\alpha_{m}}{b_{n}}\right)-f(\mathbf{x})\right) \\
\prod_{j=1}^{m}\left[\left(\begin{array}{c}
n+p \\
k_{j}
\end{array}\right) x_{j}^{k_{j}}\left(1-x_{j}\right)^{n+p-k_{j}}\right],
\end{array}
$$

then

$$
\begin{aligned}
& \left|B_{n, p}^{\left(\alpha_{1}, \ldots, \alpha_{m}\right)}(f ; \mathbf{x})-f(\mathbf{x})\right| \\
& \leq \sum_{k_{1}, \ldots, k_{m}=0}^{n+p}\left|f\left(\frac{k_{1}+\alpha_{1}}{b_{n}}, \cdots, \frac{k_{m}+\alpha_{m}}{b_{n}}\right)-f(\mathbf{x})\right| \\
& \prod_{j=1}^{m}\left[\left(\begin{array}{c}
n+p \\
k_{j}
\end{array}\right) x_{j}^{k_{j}}\left(1-x_{j}\right)^{n+p-k_{j}}\right] \\
& \leq \sum_{k_{1}, \ldots, k_{m}=0}^{n+p} \omega\left(f ; \sqrt{\sum_{i=1}^{m}\left(\frac{k_{i}+\alpha_{i}}{b_{n}}-x_{i}\right)^{2}}\right) \prod_{j=1}^{m}\left[\left(\begin{array}{c}
n+p \\
k_{j}
\end{array}\right) x_{j}^{k_{j}}\left(1-x_{j}\right)^{n+p-k_{j}}\right] \text {. }
\end{aligned}
$$

Then by using (4.1), and then applying Cauchy-Schwarz inequality, we get

$$
\begin{aligned}
& \left|B_{n, p}^{\left(\alpha_{1}, \ldots, \alpha_{m}\right)}(f ; \mathbf{x})-f(\mathbf{x})\right| \leq \omega\left(f ; \delta_{n}(x)\right) \\
& \times\left\{1+\frac{1}{\delta_{n}(x)(\mathbf{x})}\left(\sum_{k_{1}, \ldots, k_{m}=0}^{n+p} \sum_{i=1}^{m}\left(\frac{k_{i}+\alpha_{i}}{b_{n}}-x_{i}\right)^{2}\right.\right. \\
& \left.\left.\prod_{j=1}^{m}\left[\left(\begin{array}{c}
n+p \\
k_{j}
\end{array}\right) x_{j}^{k_{j}}\left(1-x_{j}\right)^{n+p-k_{j}}\right]\right)^{1 / 2}\right\} \\
& =\omega\left(f ; \delta_{n}(x)\right)+\frac{\omega\left(f ; \delta_{n}(x)\right)}{\delta_{n}(\mathbf{x})}\left(B_{n, p}^{\left(\alpha_{1}, \ldots, \alpha_{m}\right)}\left((\mathbf{t}-\mathbf{x})^{2} ; \mathbf{x}\right)\right)^{1 / 2} .
\end{aligned}
$$

By choosing

$$
\delta_{n}(\mathbf{x}):=\sqrt{B_{n, p}^{\left(\alpha_{1}, \ldots, \alpha_{m}\right)}\left((\mathbf{t}-\mathbf{x})^{2} ; \mathbf{x}\right)}
$$

then we can write the following inequality

$$
\left|B_{n, p}^{\left(\alpha_{1}, \ldots, \alpha_{m}\right)}(f ; \mathbf{x})-f(\mathbf{x})\right| \leq 2 \omega\left(f ; \sqrt{\delta_{n}(\mathbf{x})}\right) .
$$

ii) In a similar manner, we can obtain the inequality (4.2). 
Now, we want to give the quantitative result in terms of the Lipschitz class functionals. It is known that $f \in D^{p}$ belongs to $\operatorname{Lip}_{M}(\gamma)$ if

$$
|f(\mathbf{x})-f(\mathbf{y})| \leq M\left(\sum_{i=1}^{m}\left(x_{i}-y_{i}\right)^{2}\right)^{\gamma / 2}
$$

and $f \in \operatorname{Lip}_{M}\left(\gamma_{1}, \ldots, \gamma_{m}\right)$, if

$$
\left|f(\mathbf{x})-f\left(x_{1}, \ldots, x_{i-1}, y_{i}, x_{i+1, \ldots,} x_{m}\right)\right| \leq \sum_{i=1}^{m} M_{i}\left|x_{i}-y_{i}\right|^{\gamma_{i}}, i=1,2, \ldots, m
$$

where $0<\gamma \leq 1$ and $0<\gamma_{i} \leq 1(i=1,2, \ldots, m), \mathbf{x}=\left(x_{1}, \ldots, x_{m}\right), \mathbf{y}=\left(y_{1}, \ldots, y_{m}\right)$.

Theorem 4. For all $f \in \operatorname{Lip}_{M}(\gamma)$, we have

$$
\begin{aligned}
& \left|B_{n, p}^{\left(\alpha_{1}, \ldots, \alpha_{m}\right)}(f ; \mathbf{x})-f(\mathbf{x})\right| \leq M\left(\left(\left(\frac{n+p}{b_{n}}-1\right)^{2}-\frac{n+p}{b_{n}^{2}}\right)|\mathbf{x}|^{2}\right. \\
& \left.+\sum_{j=1}^{m}\left[\frac{n+p}{b_{n}^{2}}\left(2 \alpha_{j}+1\right)-2 \frac{\alpha_{j}}{b_{n}}\right] x_{j}+\frac{|\boldsymbol{\alpha}|^{2}}{b_{n}^{2}}\right)^{\gamma / 2} .
\end{aligned}
$$

Theorem 5. For all $f \in \operatorname{Lip}_{M}\left(\gamma_{1}, \ldots, \gamma_{m}\right)$, we have

$$
\begin{aligned}
& \left|B_{n, p}^{\left(\alpha_{1}, \ldots, \alpha_{m}\right)}(f ; \mathbf{x})-f(\mathbf{x})\right| \leq \sum_{i=1}^{m} M_{i}\left(\left(\left(\frac{n+p}{b_{n}}-1\right)^{2}-\frac{n+p}{b_{n}^{2}}\right) x_{i}^{2}\right. \\
& \left.+\left[\frac{n+p}{b_{n}^{2}}\left(2 \alpha_{i}+1\right)-2 \frac{\alpha_{i}}{b_{n}}\right] x_{i}+\frac{\alpha_{i}^{2}}{b_{n}^{2}}\right)^{\gamma_{i} / 2} .
\end{aligned}
$$

Remark 3. In Table 1, the error of approximation of the function $f\left(x_{1}, x_{2}\right)=$ $\cos \left(x_{1} x_{2}\right)$ by the $B_{n, p}^{\left(\alpha_{1}, \alpha_{2}\right)}\left(f ; x_{1}, x_{2}\right)$ is given, where we choose

$$
\text { alpha } a_{1}=\alpha_{2}=\frac{1}{100}, \frac{1}{90}, \frac{1}{80}, \frac{1}{70}, \frac{1}{60}
$$

and $b_{n}=1 /(\log (n+p+1)-\log (n+p))$.

TABLE 1. Error estimation table for full moduli of continuity

\begin{tabular}{|l|l|l|l|l|l|}
\hline (for $\mathrm{p}=1)$ & $n=20$ & $n=30$ & $n=50$ & $n=100$ & $n=150$ \\
\hline$x_{1}=x_{2}=0.1$ & $4.4180 \mathrm{e}-005$ & $2.8893 \mathrm{e}-005$ & $1.7007 \mathrm{e}-005$ & $8.3609 \mathrm{e}-006$ & $5.5395 \mathrm{e}-006$ \\
\hline$x_{1}=x_{2}=0.25$ & $1.5877 \mathrm{e}-004$ & $1.5877 \mathrm{e}-004$ & $1.5877 \mathrm{e}-004$ & $8.0214 \mathrm{e}-005$ & $5.3661 \mathrm{e}-005$ \\
\hline$x_{1}=x_{2}=0.5$ & $3.8730 \mathrm{e}-005$ & $2.9857 \mathrm{e}-005$ & $2.0044 \mathrm{e}-005$ & $1.0882 \mathrm{e}-005$ & $7.4546 \mathrm{e}-006$ \\
\hline$x_{1}=x_{2}=0.75$ & 0.091 & 0.0630 & 0.0039 & 0.0020 & 0.0013 \\
\hline$x_{1}=x_{2}=0.99$ & 0.0356 & 0.0246 & 0.0152 & 0.0770 & 0.0520 \\
\hline
\end{tabular}




\section{INVERSE THEOREMS}

In this section, we obtain the inverse theorems of multivariate Bernstein-SchurerStancu operators.

Theorem 6. If $f \in C_{\mathbb{R}^{m}}\left(D^{p}\right)$ such that

$$
\left|B_{n, p}^{\left(\alpha_{1}, \ldots, \alpha_{m}\right)}(f ; \mathbf{x})-f(\mathbf{x})\right| \leq M\left(\frac{1}{n+p}\right)^{\gamma}, \quad(0<\gamma \leq 1)
$$

for some positive constant $M$, then $f \in \operatorname{Li} p_{M}(\gamma)$.

Proof. For fixed $i=1,2, \ldots, m$, taking partial derivative of (2.1) with respect to $x_{i}$ we get

$$
\begin{aligned}
& \frac{\partial B_{n, p}^{\left(\alpha_{1}, \ldots, \alpha_{m}\right)}\left(f ; x_{1}, \ldots, x_{i}, \ldots, x_{m}\right)}{\partial x_{i}} \\
& =\frac{\partial}{\partial x_{i}}\left[\sum_{k_{1}, \ldots, k_{m}=0}^{n+p} f\left(\frac{k_{1}+\alpha_{1}}{b_{n}}, \cdots, \frac{k_{i}+\alpha_{i}}{b_{n}}, \cdots, \frac{k_{m}+\alpha_{m}}{b_{n}}\right)\right. \\
& \left.\times \prod_{j=1}^{m}\left[\left(\begin{array}{c}
n+p \\
k_{j}
\end{array}\right) x_{j}^{k_{j}}\left(1-x_{j}\right)^{n+p-k_{j}}\right]\right] \\
& =\frac{\partial}{\partial x_{i}}\left[\sum_{k_{1}, \ldots, k_{m}=0}^{n+p} f\left(\frac{k_{1}+\alpha_{1}}{b_{n}}, \cdots, \frac{k_{i}+\alpha_{i}}{b_{n}}, \cdots, \frac{k_{m}+\alpha_{m}}{b_{n}}\right)\right. \\
& \left.\times\left[\left(\begin{array}{c}
n+p \\
k_{i}
\end{array}\right) x_{i}^{k_{i}}\left(1-x_{i}\right)^{n+p-k_{i}}\right] \prod_{\substack{j=1 \\
j \neq i}}^{m}\left[\left(\begin{array}{c}
n+p \\
k_{j}
\end{array}\right) x_{j}^{k_{j}}\left(1-x_{j}\right)^{n+p-k_{j}}\right]\right] \\
& =\sum_{k_{i}=1}^{n+p} \sum_{k_{1}, \ldots, k_{i-1}, k_{i+1} \ldots, k_{m}=0}^{n+p} f\left(\frac{k_{1}+\alpha_{1}}{b_{n}}, \cdots, \frac{k_{i}+\alpha_{i}}{b_{n}}, \cdots, \frac{k_{m}+\alpha_{m}}{b_{n}}\right) \\
& \times \frac{(n+p) !}{\left(k_{i}-1\right) !\left(n+p-k_{i}\right) !} x_{i}^{k_{i-1}}\left(1-x_{i}\right)^{n+p-k_{i}} \prod_{\substack{j=1 \\
j \neq i}}^{m}\left[\left(\begin{array}{c}
n+p \\
k_{j}
\end{array}\right) x_{j}^{k_{j}}\left(1-x_{j}\right)^{n+p-k_{j}}\right] \\
& -\sum_{k_{i}=0}^{n+p-1} \sum_{k_{1}, \ldots, k_{i-1}, k_{i+1} \ldots, k_{m}=0}^{n+p} f\left(\frac{k_{1}+\alpha_{1}}{b_{n}}, \cdots, \frac{k_{i}+\alpha_{i}}{b_{n}}, \cdots, \frac{k_{m}+\alpha_{m}}{b_{n}}\right)
\end{aligned}
$$




$$
\begin{aligned}
& \times \frac{(n+p) !}{k_{i} !\left(n+p-k_{i}-1\right) !} x_{i}^{k_{i}}\left(1-x_{i}\right)^{n+p-k_{i}-1} \prod_{\substack{j=1 \\
j \neq i}}^{m}\left[\left(\begin{array}{c}
n+p \\
k_{j}
\end{array}\right) x_{j}^{k_{j}}\left(1-x_{j}\right)^{n+p-k_{j}}\right] \\
& =\sum_{k_{i}=0 \quad k_{1}, \ldots, k_{i-1}, k_{i+1} \ldots, k_{m}=0}^{n+p-1} f\left(\frac{k_{1}+\alpha_{1}}{b_{n}}, \cdots, \frac{k_{i}+1+\alpha_{i}}{b_{n}}, \cdots, \frac{k_{m}+\alpha_{m}}{b_{n}}\right) \\
& \times \frac{(n+p)(n+p-1) !}{k_{i} !\left(n+p-k_{i}-1\right) !} x_{i}^{k_{i}}\left(1-x_{i}\right)^{n+p_{i}-k_{i}-1} \prod_{\substack{j=1 \\
j \neq i}}^{m}\left[\left(\begin{array}{c}
n+p_{j} \\
k_{j}
\end{array}\right) x_{j}^{k_{j}}\left(1-x_{j}\right)^{n+p_{j}-k_{j}}\right] \\
& -\sum_{k_{i}=0 \quad k_{1}, \ldots, k_{i-1}, k_{i+1} \ldots, k_{m}=0}^{n+p} f\left(\frac{k_{1}+\alpha_{1}}{b_{n}}, \cdots, \frac{k_{i}+\alpha_{i}}{b_{n}}, \cdots, \frac{k_{m}+\alpha_{m}}{b_{n}}\right) \\
& \times \frac{(n+p)(n+p-1) !}{k_{i} !\left(n+p-k_{i}-1\right) !} x_{i}^{k_{i}}\left(1-x_{i}\right)^{n+p_{i}-k_{i}-1} \prod_{\substack{j=1 \\
j \neq i}}^{m}\left[\left(\begin{array}{c}
n+p \\
k_{j}
\end{array}\right) x_{j}^{k_{j}}\left(1-x_{j}\right)^{n+p-k_{j}}\right] .
\end{aligned}
$$

Now, we get

$$
\begin{gathered}
\frac{\partial B_{n, p}^{\left(\alpha_{1}, \ldots, \alpha_{m}\right)}(f ; \mathbf{x})}{\partial x_{i}} \\
=(n+p) \sum_{k_{i}=0}^{n+p-1} \sum_{k_{1}, \ldots, k_{i-1}, k_{i+1} \ldots, k_{m}=0}^{n+p} \\
\quad\left(f\left(\frac{k_{1}+\alpha_{1}}{b_{n}}, \cdots, \frac{k_{i}+1+\alpha_{i}}{b_{n}}, \cdots, \frac{k_{m}+\alpha_{m}}{b_{n}}\right)\right. \\
\left.\quad-f\left(\frac{k_{1}+\alpha_{1}}{b_{n}}, \cdots, \frac{k_{i}+\alpha_{i}}{b_{n}}, \cdots, \frac{k_{m}+\alpha_{m}}{b_{n}}\right)\right) \\
\times \frac{(n+p-1) !}{k_{i} !\left(n+p-k_{i}-1\right) !} x_{i}^{k_{i}}\left(1-x_{i}\right)^{n+p-k_{i}-1} \prod_{\substack{j=1 \\
j \neq i}}^{m}\left[\left(\begin{array}{c}
n+p \\
k_{j}
\end{array}\right) x_{j}^{k_{j}}\left(1-x_{j}\right)^{n+p-k_{j}}\right] .
\end{gathered}
$$

Then, by triangle inequality,

$$
\begin{aligned}
& \left|\frac{\partial B_{n, p}^{\left(\alpha_{1}, \ldots, \alpha_{m}\right)}(f ; \mathbf{x})}{\partial x_{i}}\right| \\
& \leq(n+p) \sum_{k_{i}=0}^{n+p-1} \sum_{k_{1}, \ldots, k_{i-1}, k_{i+1}, \ldots, k_{m}=0}^{n+p}
\end{aligned}
$$




$$
\begin{gathered}
\mid f\left(\frac{k_{1}+\alpha_{1}}{b_{n}}, \cdots, \frac{k_{i}+1+\alpha_{i}}{b_{n}}, \cdots, \frac{k_{m}+\alpha_{m}}{b_{n}}\right) \\
-f\left(\frac{k_{1}+\alpha_{1}}{b_{n}}, \cdots, \frac{k_{i}+\alpha_{i}}{b_{n}}, \cdots, \frac{k_{m}+\alpha_{m}}{b_{n}}\right) \mid \\
\times \frac{(n+p-1) !}{k_{i} !\left(n+p-k_{i}-1\right) !} x_{i}^{k_{i}}\left(1-x_{i}\right)^{n+p-k_{i}-1} \prod_{\substack{j=1 \\
j \neq i}}^{m}\left[\left(\begin{array}{c}
n+p \\
k_{j}
\end{array}\right) x_{j}^{k_{j}}\left(1-x_{j}\right)^{n+p-k_{j}}\right] .
\end{gathered}
$$

Using (4.2), we have

$$
\begin{aligned}
& \left|\frac{\partial B_{n, p}^{\left(\alpha_{1}, \ldots, \alpha_{m}\right)}(f ; \mathbf{x})}{\partial x_{i}}\right| \\
& \leq(n+p) \sum_{k_{i}=0}^{n+p-1} \sum_{k_{1}, \ldots, k_{i-1}, k_{i+1} \ldots, k_{m}=0}^{n+p} \omega^{(i)}\left(f ; \frac{1}{b_{n}}\right) \frac{(n+p-1) !}{k_{i} !\left(n+p-k_{i}-1\right) !} \\
& \times x_{i}^{k_{i}}\left(1-x_{i}\right)^{n+p-k_{i}-1} \prod_{\substack{j=1 \\
j \neq i}}^{m}\left[\left(\begin{array}{c}
n+p \\
k_{j}
\end{array}\right) x_{j}^{k_{j}}\left(1-x_{j}\right)^{n+p-k_{j}}\right] \\
& =(n+p) \sum_{k_{i}=0}^{n+p-1} \omega^{(i)}\left(f ; \frac{1}{b_{n}}\right) \frac{(n+p-1) !}{k_{i} !\left(n+p-k_{i}-1\right) !} x_{i}^{k_{i}}\left(1-x_{i}\right)^{n+p-k_{i}-1} \\
& \leq(n+p) \omega^{(i)}(f ; \delta) \sum_{k_{i}=0}^{n+p-1} \\
& \left(\left(1+\frac{1}{\delta b_{n}}\right) \frac{(n+p-1) !}{k_{i} !\left(n+p-k_{i}-1\right) !} x_{i}^{k_{i}}\left(1-x_{i}\right)^{n+p-k_{i}-1}\right) \\
& =(n+p) \omega^{(i)}(f ; \delta)\left\{1+\frac{1}{\delta b_{n}}\right\}=\omega^{(i)}(f ; \delta)\left\{n+p+\frac{n+p}{\delta b_{n}}\right\} \text {. }
\end{aligned}
$$

For any pair of points $x, y$ in $[0,1]$, we can write

$$
\begin{aligned}
& \int_{x}^{y}\left|\frac{\partial B_{n, p}^{\left(\alpha_{1}, \ldots, \alpha_{m}\right)}(f ; \mathbf{x})}{\partial x_{i}}\right| d x_{i} \leq \quad \omega^{(i)}(f ; \delta)\left\{n+p+\frac{n+p}{\delta b_{n}}\right\} \int_{x}^{y} d x_{i} \\
& \int_{x}^{y}\left|\frac{\partial B_{n, p}^{\left(\alpha_{1}, \ldots, \alpha_{m}\right)}(f ; \mathbf{x})}{\partial x_{i}}\right| d x_{i} \leq \quad \omega^{(i)}(f ; \delta)|x-y|\left\{n+p+\frac{n+p}{\delta b_{n}}\right\} .
\end{aligned}
$$


By choosing $\delta_{n}:=\frac{1}{n+p}$, we obtain

$$
\int_{x}^{y}\left|\frac{\partial B_{n, p}^{\left(\alpha_{1}, \ldots, \alpha_{m}\right)}(f ; \mathbf{x})}{\partial x_{i}}\right| d x_{i} \leq \omega^{(i)}(f ; \delta)|x-y|\left\{\frac{1}{\delta_{n}}+\frac{n+p}{\delta b_{n}}\right\} .
$$

Then the sequences $\delta_{n}$ decreases to zero as $n \rightarrow \infty$. Also $\delta_{n-1} \leq 2 \delta_{n}$ is satisfied for $n=2,3, \cdots$. Hence for a given $0<\delta \leq 1$ there exists $n \in \mathbb{N}$ such that

$$
\delta_{n} \leq \delta \leq \delta_{n-1} \leq 2 \delta_{n} \text {. }
$$

Using (5.2) in (5.1), we have

$$
\int_{x}^{y}\left|\frac{\partial B_{n, p}^{\left(\alpha_{1}, \ldots, \alpha_{m}\right)}(f ; \mathbf{x})}{\partial x_{i}}\right| d x_{i} \leq\left\{2+\frac{n+p}{b_{n}}\right\} \frac{1}{\delta} \omega^{(i)}(f ; \delta)|x-y| .
$$

On the other hand, since we have

$$
\begin{aligned}
& |f(\mathbf{x})-f(\mathbf{y})| \leq\left|f(\mathbf{x})-f\left(y_{1}, x_{2}, \ldots, x_{m}\right)\right| \\
& +\left|f\left(y_{1}, x_{2}, \ldots, x_{m}\right)-f\left(y_{1}, y_{2}, \ldots, x_{m}\right)\right|+\cdots \\
& +\left|f\left(y_{1}, \ldots, y_{m-1}, \ldots, x_{m}\right)-f(\mathbf{y})\right|
\end{aligned}
$$

and

$$
\begin{aligned}
& \left|f(\mathbf{x})-f\left(y_{1}, x_{2}, \ldots, x_{m}\right)\right| \leq\left|f(\mathbf{x})-B_{n, p}^{\left(\alpha_{1}, \ldots, \alpha_{m}\right)}(f ; \mathbf{x})\right| \\
& +\left|f\left(y_{1}, x_{2}, \ldots, x_{m}\right)-B_{n, p}^{\left(\alpha_{1}, \ldots, \alpha_{m}\right)}\left(f ; y_{1}, x_{2}, \ldots, x_{m}\right)\right| \\
& +\int_{x_{1}}^{y_{1}}\left|\frac{\partial}{\partial x} B_{n, p}^{\left(\alpha_{1}, \ldots, \alpha_{m}\right)}\left(f ; x, x_{2}, \ldots, x_{m}\right)\right| d x, \\
& \left|f\left(y_{1}, x_{2}, \ldots, x_{m}\right)-f\left(y_{1}, y_{2}, x_{3} \ldots, x_{m}\right)\right| \\
& \leq\left|f\left(y_{1}, x_{2}, \ldots, x_{m}\right)-B_{n, p}^{\left(\alpha_{1}, \ldots, \alpha_{m}\right)}\left(f ; y_{1}, x_{2}, \ldots, x_{m}\right)\right| \\
& +\left|f\left(y_{1}, y_{2}, \ldots, x_{m}\right)-B_{n, p}^{\left(\alpha_{1}, \ldots, \alpha_{m}\right)}\left(f ; y_{1}, y_{2}, \ldots, x_{m}\right)\right| \\
& \quad+\int_{x_{2}}^{y_{2}}\left|\frac{\partial}{\partial x} B_{n, p}^{\left(\alpha_{1}, \ldots, \alpha_{m}\right)}\left(f ; y_{1}, x, \ldots, x_{m}\right)\right| d x
\end{aligned}
$$

$\vdots$

$$
\begin{aligned}
& \left|f\left(y_{1}, y_{2}, \ldots, x_{m}\right)-f\left(y_{1}, y_{2}, \ldots, y_{m}\right)\right| \\
& \leq\left|f\left(y_{1}, \ldots, y_{m-1}, x_{m}\right)-B_{n, p}^{\left(\alpha_{1}, \ldots, \alpha_{m}\right)}\left(f ; y_{1}, \ldots, y_{m-1}, x_{m}\right)\right| \\
& +\left|f\left(y_{1}, y_{2}, \ldots, y_{m}\right)-B_{n, p}^{\left(\alpha_{1}, \ldots, \alpha_{m}\right)}\left(f ; y_{1}, y_{2}, \ldots, y_{m}\right)\right|
\end{aligned}
$$




$$
+\int_{x_{m}}^{y_{m}}\left|\frac{\partial}{\partial x} B_{n, p}^{\left(\alpha_{1}, \ldots, \alpha_{m}\right)}\left(f ; y_{1}, y_{2}, \ldots, x\right)\right| d x
$$

we get, for all fixed $n \in \mathbb{N}$

$$
\begin{aligned}
& \left|f(\mathbf{x})-f\left(y_{1}, \ldots, y_{m}\right)\right| \\
& \leq\left|f(\mathbf{x})-B_{n, p}^{\left(\alpha_{1}, \ldots, \alpha_{m}\right)}(f ; \mathbf{x})\right| \\
& +\left|f\left(y_{1}, x_{2}, \ldots, x_{m}\right)-B_{n, p}^{\left(\alpha_{1}, \ldots, \alpha_{m}\right)}\left(f ; y_{1}, x_{2}, \ldots, x_{m}\right)\right| \\
& +\left|f\left(y_{1}, x_{2}, \ldots, x_{m}\right)-B_{n, p}^{\left(\alpha_{1}, \ldots, \alpha_{m}\right)}\left(f ; y_{1}, x_{2}, \ldots, x_{m}\right)\right| \\
& +\left|f\left(y_{1}, y_{2}, \ldots, x_{m}\right)-B_{n, p}^{\left(\alpha_{1}, \ldots, \alpha_{m}\right)}\left(f ; y_{1}, y_{2}, \ldots, x_{m}\right)\right| \\
& +\cdots+\left|f\left(y_{1}, \ldots, y_{m-1}, y_{m}\right)-B_{n, p}^{\left(\alpha_{1}, \ldots, \alpha_{m}\right)}\left(f ; y_{1}, \ldots, y_{m}\right)\right| \\
& \quad+\int_{x_{1}}^{y_{1}}\left|\frac{\partial}{\partial x} B_{n, p}^{\left(\alpha_{1}, \ldots, \alpha_{m}\right)}\left(f ; x, x_{2} \ldots, x_{m}\right)\right| d x \\
& \quad+\int_{x_{2}}^{y_{2}}\left|\frac{\partial}{\partial x} B_{n, p}^{\left(\alpha_{1}, \ldots, \alpha_{m}\right)}\left(f ; y_{1}, x, \ldots, x_{m}\right)\right| d x \\
& +\cdots+\int_{x_{m}}^{y_{m}}\left|\frac{\partial}{\partial x} B_{n, p}^{\left(\alpha_{1}, \ldots, \alpha_{m}\right)}\left(f ; y_{1}, y_{2}, \ldots, x\right)\right| d x .
\end{aligned}
$$

Using (5.2) and (5.3) in (5.5), we obtain

$$
\begin{aligned}
& |f(\mathbf{x})-f(\mathbf{y})| \leq 2 m M \delta^{\gamma}+\sum_{i=1}^{m}\left(2+\frac{n+p}{b_{n}}\right) \frac{1}{\delta} \omega^{(i)}(f ; \delta)\left(\sum_{j=1}^{m}\left(x_{j}-y_{j}\right)^{2}\right)^{1 / 2}, \\
& \leq 2 m M \delta^{\gamma}+\left(2+\frac{n+p}{b_{n}}\right) \frac{m}{\delta} \omega(f ; \delta)\left(\sum_{j=1}^{m}\left(x_{j}-y_{j}\right)^{2}\right)^{1 / 2} \\
& \leq K_{1}\left(\delta^{\gamma}+\frac{\omega(f ; \delta)}{\delta}\left(\sum_{j=1}^{m}\left(x_{j}-y_{j}\right)^{2}\right)^{1 / 2}\right)
\end{aligned}
$$

where $K_{1}=\max \left\{2 m M, m\left(2+\frac{n+p}{b_{n}}\right)\right\}$.

Therefore, taking $\left(\sum_{j=1}^{m}\left(x_{j}-y_{j}\right)^{2}\right)^{1 / 2} \leq h$ with $0<h \leq 1$ and considering the definition of $\omega$, we write

$$
\omega(f ; h) \leq K_{1}\left(\delta^{\gamma}+\frac{h}{\delta} \omega(f ; \delta)\right), \quad 0<h, \delta \leq 1 .
$$


Then, by (5.6) we have $K_{2} h^{\gamma}$. Hence, we get

$$
\omega(f ; h) \leq K_{2} h^{\gamma} \quad(0<\gamma<1) .
$$

From (5.7), we obtain $f \in L i p \gamma$. We get the desired result.

In a similar manner, we can state the following theorem:

Theorem 7. If the inequality

$$
\left|B_{n, p}^{\left(\alpha_{1}, \ldots, \alpha_{m}\right)}(f ; \mathbf{x})-f\left(x_{1}, \ldots, x_{j-1}, y_{j}, x_{j+1}, \ldots, x_{m}\right)\right| \leq M_{j}\left(\frac{1}{n+p}\right)^{\gamma_{j}}
$$

is satisfied for $0<\gamma_{j} \leq 1$ and for some constants $M_{j},(j=1,2, \ldots, m)$, then

$$
f \in \bigcap_{j=1}^{m} \operatorname{Lip}_{M_{j}} \gamma_{j}
$$

\section{REFERENCES}

[1] D. Barbosu, "Schurer-Stancu type operators," Studia Univ. 'Babeş Bolyai' Mathematica, vol. 48, no. 3, pp. 31-35, 2003.

[2] D. Barbosu, "Bivariate operators of Schurer-Stancu type," Rev. Anal. Numer. et de Ther. de l' Approx., vol. 34, no. 1, pp. 17-21, 2005.

[3] D. Barbosu, "Simultaneous approximation by bivariate Schurer-Stancu type operators," Math Balkanica, vol. 20, no. 3-4, pp. 351-358, 2006.

[4] P. L. Butzer, "On two dimensional Bernstein polynomials," Canad. J. Math., vol. 5, pp. 107-113, 1953, doi: 10.4153/CJM-1953-014-2.

[5] I. Buyukyazici and E. Ibikli, "The approximation properties of generalized Bernstein polynomials of two variables," Journal of Compt. and Appl. Mathematics, vol. 156, pp. 367-380, 2004, doi: 10.1016/j.amc.2003.07.023.

[6] I. Buyukyazici and E. Ibikli, "Direct and converse results for multivariate generalized Bernstein polynomials," Journal of Compt. and Appl. Mathematics, vol. 219, no. 219, pp. 145-155, 2008, doi: 10.1016/j.cam.2007.06.035.

[7] F. Cao, "Derivatives of multidimensional Bernstein operators and smothness," J. Approx. Theory, vol. 132, pp. 241-257, 2005, doi: 10.1016/j.jat.2004.12.006.

[8] P. N. Chak, N. Madras, and B. Smith, "Semi-nonparametric estimation with Bernstein polynomials," Economics Letter, vol. 89, pp. 153-156, 2005, doi: 10.1016/j.econlet.2005.01.025.

[9] Z. Ditzian, "Inverse theorems for multidimensional Bernstein operators," Pasific J. Math., vol. 121, no. 2, pp. 293-319, 1986, doi: 10.2140/pjm.1986.121.293.

[10] Z. Ditzian and D. Jiang, "Inverse theorems for best approximation in C[0,1]," Proc. Amer. Math. Soc., vol. 120, no. 151-155, pp. 300-306, 1994.

[11] H. Gonska, M. Heilmann, and I. Rasa, "Asymtotic behaviour of differentiated Bernstein polynomials revisited," General Mathematics (Sibiu), vol. 18, pp. 45-53, 2010.

[12] H. Gonska, M. Heilmann, and I. Rasa, "Kantorovich operators of order k," Numer. Funct. Anal. Optimiz., vol. 32, pp. 717-738, 2011, doi: 10.1080/01630563.2011.580877.

[13] H. Haciyev and H. H. Hacisalihoglu, Lineer Pozitif Operatorlerin Yakinsakligi. Ankara: A.O.F.F. Doner Sermaye Isletmesi Yayinlari, 1995.

[14] H. S. Jung, N. Deo, and M. Dhamija, "Pointwise approximation by Bernstein type operators in mobile interval," Appl. Math. Comput., vol. 214, no. 1, pp. 683-694, 2014, doi: 10.1016/j.amc.2014.07.034. 
[15] Z. Li, "Bernstein polynomials and modulus of continuity," Journal of Approximation Theory, vol. 102, pp. 171-174, 2000, doi: 10.1006/jath.1999.3374.

[16] G. G. Lorentz, Bernstein Polynomials, 2nd ed. New York: Chelsea Publishing Co, 1986.

[17] G. G. Lorentz and R. A. DeVore, Constructive Approximation. Berlin: Springer-Verlag, 1993.

[18] L. Martinez, "Some properties of two-dimensional Bernstein polynomials," J. Approx. Theory, vol. 5, pp. 300-306, 1989, doi: 10.1016/0021-9045(89)90095-6.

[19] C. V. Muraru, "Note on the q-Stancu-Schurer operator," Miskolc Mathematical Notes, vol. 14, no. 1, pp. 191-200, 2003.

[20] G. M. Phillips, On Generalized Bernstein Polynomials. River Edge: World Sci. Publ., 1996.

[21] G. M. Phillips, Interpolation and Approximation by Bernstein Polynomials, ser. CMS Books in Mathematics. New York: Springer-Verlag, 2003, vol. 14, doi: 10.1007/b97417.

[22] F. Schurer, "Linear positive operators in approximation theory," Math. Inst., Techn. Univ. Delf Report, pp. 263-269, 1962.

[23] V. E. Wickeren, "Direct and inverse theorems for Bernstein polynomials in the space of Riemann integrable functions," Constr. Approx., vol. 5, pp. 189-198, 1989, doi: 10.1007/BF01889606.

Authors' addresses

Mehmet Ali Özarslan

Eastern Mediterranean University, Gazimagusa, TRNC, Mersin 10, Turkey

E-mail address: mehmetali.ozarslandemu.edu.tr

Tuba Vedi

Eastern Mediterranean University, Gazimagusa, TRNC, Mersin 10, Turkey

E-mail address: tuba.vedi@emu.edu.tr 